\title{
Integrated APT/t-EBSD for Grain Boundary Analysis of Thermally Grown Oxide on a Ni-Based Superalloy
}

\author{
Y. Chen ${ }^{1}$, K.P. Rice ${ }^{1}$, T.J. Prosa ${ }^{1}$, E.A. Marquis ${ }^{2}$ and R.C. Reed $^{3}$ \\ 1. CAMECA Instruments, Inc., Madison, WI, USA \\ 2. Dept. of Materials Science and Engineering, University of Michigan, Ann Arbor, MI, USA \\ 3. Dept. of Engineering Science, University of Oxford, Parks Road, Oxford, OX1 3PG, UK
}

Thermal barrier coatings (TBCs) allow turbine engines to be operated at temperatures greater than the melting temperatures of engine components to pursue better propulsive power performance and fuel efficiency [1]. TBCs generally consist of three layers. As illustrated in Figure 1, on the top is a coat made of yttrium-stabilized $\mathrm{ZrO}_{2}$ (or YSZ) which has excellent thermal resistivity. Beneath the YSZ layer is a thermally grown oxide (TGO) scale that consists of $\alpha$-alumina grains. At the coating/substrate interface is a bond coat layer that improves adhesion of the ceramic layers on the superalloy substrate.

The TGO layer typically forms as a thin layer during deposition of the YSZ layer and grows during service. The growth of the TGO introduces additional interfacial stresses to the coating that can eventually lead to delamination of the TBC structure. A longer TBC life-time is achieved by slowing down the growth of the TGO layer. In the case of $\alpha$-alumina, the inward diffusion of oxygen along grain boundaries is dominant mechanism for the oxide growth. Alloying with reactive elements (REs), such as Y, Hf, La, has been shown to significantly reduce the growth rate of TGO, and while these elements have been found to segregate to grain boundaries, the exact phenomenon by which oxygen transport is affected is not clear. Atom probe tomography (APT) was previously used to quantify grain boundary chemistry in TGO layers. Significant chemical variations between different grain boundaries were reported, suggesting a strong effect of grain boundary character [2]. Inspired by the ability of mapping needle shaped specimens using electron backscatter diffraction (EBSD) in transmission mode, this work aims to further investigate the relationships between grain boundary chemistry and grain boundary misorientation using the integrated APT/t-EBSD technique [3]. Linking orientation to chemistry will inform on the possible pathways to controlling alumina growth.

Mo grids were cut in half and electropolished for mounting specimens. Needle-shaped APT specimens were prepared by a standard lift-out method and annular milling using an FEI Nova FIB/SEM. The integrated EXAX EBSD system allows for in-situ mapping feedback between milling processes. Figure 2 is a typical t-EBSD pattern from an $\alpha$-alumina grain in TGO collected at a tilt angle of 155 degrees using $30 \mathrm{kV}$ electrons. Figure $3 \mathrm{a}$ is a t-EBSD map showing multiple grains in an APT specimen for targeting grain boundaries. Two separate APT analyses were performed in region $\mathbf{A}$ and $\mathbf{B}$ in Figure $3 \mathrm{a}$. In region $\mathbf{A}$, grain boundaries segregated with $\mathrm{Zr}$ are visible and the grain misorientation of the two alumina grains on the bottom were subsequently measured using t-EBSD (Figure 3c) by terminating the APT analysis before tip fracture. The same tip was further FIB milled into region $\mathbf{B}$ and mapped with tEBSD using a step size of $10 \mathrm{~nm}$ (Figure 3d). Two grain boundaries were successfully captured by APT (Figure $3 \mathrm{e}$ ) and their structures and chemistries can be studies accordingly.

In this study we have demonstrated that high-resolution t-EBSD maps can be acquired on needle-shaped APT specimens that consist of alumina grains of size ranging from few hundred nanometers to few micrometers. Transmission EBSD mapping offers the ability to target site-specific grains for APT 
analysis, and correlate grain boundary chemistries with grain misorientations. In addition, we succeeded in collecting multiple APT datasets and t-EBSD maps from a single APT specimen, thus the evolution of structure across TGO scales can be investigated.

\section{References:}

[1] A. Evans et al., Prog Mater Sci 46 (2001), p. 505.

[2] Y Chen et al., Oxidation of Metals 82 (2014), p. 457.

[3] K. Babinsky et al., Ultramicroscopy 144 (2014), p. 9.

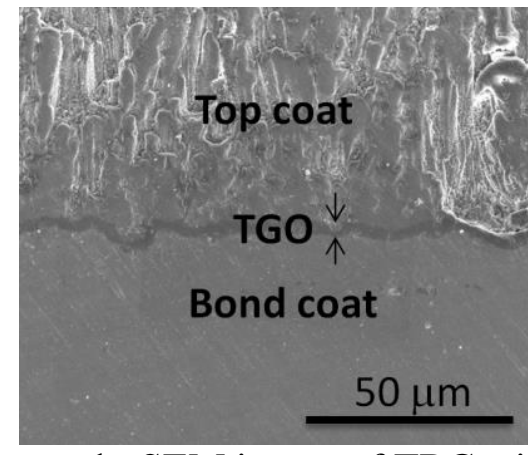

Figure 1. SEM image of TBC with a $3 \mu \mathrm{m}$ thick TGO scale on a Ni-based superalloy

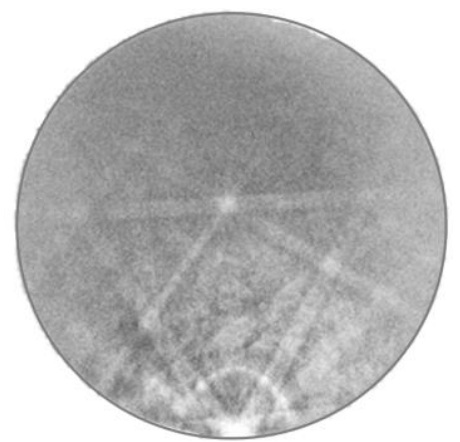

Figure 2. A typical EBSD pattern of $\alpha$-alumina collected with $30 \mathrm{kV}$ electrons

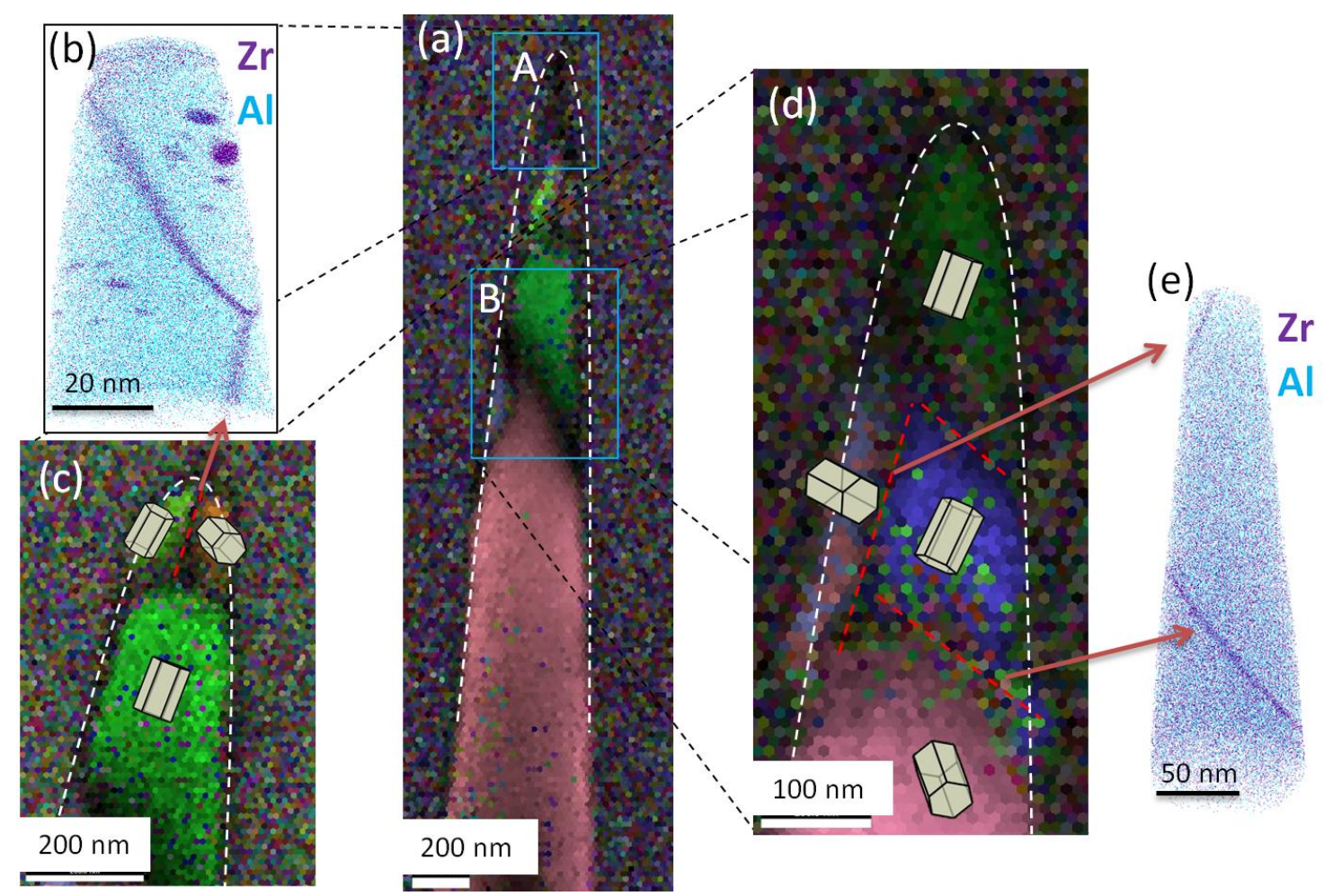

Figure 3. t-EBSD maps of alumina grains and correlative APT results from region $\mathbf{A}$ and 